\title{
Hipoacusia unilateral: bases neurobiológicas de la ambliaudia
}

\section{Unilateral hearing loss: neurobiological basis of amblyaudia}

\author{
Sergio Vicencio J. ${ }^{1,2,3}$, Mariela Torrente A. ${ }^{4,5}$, Jonathan Wimmer del Solar ${ }^{1,7}$, Paul Délano R. ${ }^{1,2,4,6}$
}

'Laboratorio de Neurobiología de la Audición, Departamento de Neurociencia, Facultad de Medicina, Universidad de Chile. Santiago, Chile. ${ }^{2}$ Biomedical Neuroscience Institute, BNI, Facultad de Medicina, Universidad de Chile. Santiago, Chile.

${ }^{3}$ Center for Hearing and Balance and

Department of Otolaryngology-HNS, Johns Hopkins University. Baltimore, USA. ${ }^{4}$ Departamento de Otorrinolaringología, Hospital Clínico Universidad de Chile. Santiago, Chile.

${ }^{5}$ Servicio de Otorrinolaringología, Hospital Padre Hurtado. Santiago, Chile.

${ }^{6}$ Centro Avanzado de Ingeniería Eléctrica y Electrónica, AC3E, Universidad Técnica Federico Santa María. Valparaíso, Chile. Programa de Neurología, Facultad de Ciencias Médicas, Universidad de Santiago de Chile. Santiago, Chile.

Los autores declaran no tener conflictos de interés. Paul Délano R. es financiado por Fondo Basal FB0008, ANID; Iniciativa Científica Milenio, Proyecto ICM P09-015F; y Fundación Guillermo Puelma.

Recibido el 4 de diciembre de 2019. Aceptado el 11 de abril de 2020.

Correspondencia: Sergio Vicencio J. Independencia 1027

Departamento de Neurociencia, Facultad de Medicina, Universidad de Chile. Santiago, Chile. Email: vicenciojiménez@gmail.com

\section{Resumen}

La hipoacusia unilateral es una alteración prevalente e importante en la población infantil que puede llevar a un deterioro del procesamiento auditivo. Cualquier patología que cause hipoacusia asimétrica (conductiva o sensorioneural) durante ventanas críticas del desarrollo cerebral, puede dar lugar a déficits del procesamiento auditivo que podrían persistir incluso después de la rehabilitación audiológica. Este proceso fisiopatológico se ha denominado "ambliaudia" y los niños que la presentan tienen un mayor riesgo de sufrir retraso en el desarrollo del lenguaje, así como de padecer dificultades académicas, sociales y cognitivas. Por esto, la ambliaudia se presenta como un desafío en audiología, siendo necesario comprender los mecanismos neurobiológicos que la subyacen. Entender de mejor manera cuáles son las consecuencias que tiene la pérdida auditiva asimétrica sobre el desarrollo del sistema nervioso podría ser clave para guiar de mejor forma las intervenciones terapéuticas. En este artículo se revisan trabajos en humanos y en modelos animales que describen las consecuencias neurobiológicas de la deprivación auditiva unilateral durante el desarrollo del sistema nervioso y su posible aplicación a la práctica clínica. Palabras clave: hipoacusia unilateral, procesamiento auditivo, ambliaudia, vías auditivas.

\begin{abstract}
Unilateral hearing loss is a prevalent and important disorder in children that can lead to an impairment of auditory processing. Any pathology that causes asymmetric (conductive or sensorineural) hearing loss during critical brain development windows can lead to hearing deficits that may persist even after audiological rehabilitation. This pathophysiological process has been referred to as amblyaudia, and children are at greater risk of experiencing language developmental delays as well as academic, social and cognitive impairments. For this reason, amblyaudia presents itself as a challenge in audiology, and it has become necessary to understand its neurobiological mechanisms. A better understanding of the consequences of asymmetric hearing loss on the development of the nervous system may be key to better guiding therapeutic interventions. This article reviews studies performed in humans and animal models that describe the neurobiological consequences of unilateral hearing deprivation during the development of the nervous system and its possible applications to clinical practice.
\end{abstract}

Keywords: unilateral hearing loss, auditory processing, amblyaudia, auditory pathways alterations.

\section{Introducción}

La hipoacusia es un diagnóstico relevante en la población infantil. Su incidencia al nacer fluctúa entre 0,5 a 3 casos por cada 1.000 recién nacidos $^{1-3}$. Más aún, esta cifra se incrementa en niños de edad escolar, estimándose una ocurrencia entre 4 a 10 de cada 1.000 niños $^{4,5}$. Dentro de este universo, la pérdida auditiva unilateral suele pasar desapercibida como un 
problema de salud importante, pese que hasta el $12 \%$ de los niños menores de tres años pueden llegar a experimentar una pérdida auditiva unilateral temporal ${ }^{6}$. Un niño presenta pérdida auditiva unilateral cuando manifiesta algún grado de disfunción auditiva en un oído, mientras que su otro oído tiene un nivel normal de audición. Las razones de que esta condición suela pasar por alto son multifactoriales. En parte, se pueden explicar debido a que esta condición suele no ser detectada por los adultos responsables, los propios niños, o incluso por los profesionales de la salud. Además, de los casos identificados, algunos se pierden durante el seguimiento o se tratan de forma insuficiente $\mathrm{e}^{7-9}$. Esta falta de tratamiento, en algunos casos se debe a la idea de que el oído contralateral con audición normal puede compensar una pérdida auditiva unilateral ${ }^{8,10}$. Sin embargo, hace ya más de treinta años que existe evidencia de que la hipoacusia unilateral no resulta inocua. Los niños con pérdida auditiva unilateral tienen un mayor riesgo de sufrir retraso en el desarrollo del habla y del lenguaje, así como de padecer dificultades académicas, sociales y cognitivas ${ }^{11-15}$. Es por estas consecuencias que en la actualidad es cada vez más común que la pérdida auditiva asimétrica sea tratada mediante amplificación o intervención quirúrgica ${ }^{16}$.

\section{Relevancia de la audición biaural}

El correcto funcionamiento del sistema auditivo depende de la integración de señales sensoriales bilaterales, que resultan clave para el procesamiento auditivo de alta complejidad. Esta información bilateral permite identificar sonidos de interés en ambientes ruidosos y es necesaria para poder transformar las señales neuronales unidimensionales en una representación tridimensional del espacio auditivo ${ }^{16,17}$. Junto a esto, existe evidencia que el desarrollo del sistema auditivo se ve afectado por estímulos ambientales, por lo que si este se da bajo condiciones asimétricas puede llegar a provocar alteraciones anatómicas y funcionales permanentes en el sistema nervioso central ${ }^{18}$. En efecto, estudios en modelos animales y humanos han mostrado evidencia de que, si la hipoacusia unilateral no es tratada dentro de una ventana de tiempo crítico, las alteraciones del procesamiento auditivo sufridos en edad temprana pueden persistir incluso después de que se haya resuelto la pérdida auditiva unilateral $^{6,19}$. Por ejemplo, en roedores se ha observado que la hipoacusia unilateral conductiva tiene como consecuencia la aparición de un desbalance entre el lado comprometido y el sano, disminuyendo la representación neuronal del oído afectado y llevando a la generación de un lado dominante en los niveles de mayor jerarquía de la vía auditiva (como la corteza auditiva primaria $)^{19}$. En humanos, luego de la corrección quirúrgica de la pérdida auditiva unilateral congénita conductiva, los pacientes suelen presentar déficits duraderos en la audición binaural (especialmente en tareas como localización de sonidos y comprensión del habla) a pesar de presentar audiogramas prácticamente normales ${ }^{20}$. Estos tipos de hallazgos se han visto asociados con la edad de intervención quirúrgica, especialmente si esta se retrasa hasta después de los 5 años $^{21}$. También, se ha descrito que los niños con hipoacusia sensorioneural unilateral que son tratados precozmente con un audífono presentan mejor procesamiento de señales auditivas bilaterales ${ }^{22}$.

Las alteraciones del procesamiento auditivo bilateral a largo plazo, que surgen como consecuencia de una hipoacusia unilateral ocurrida durante un período crítico del desarrollo del sistema nervioso han sido definidas como ambliaudia ${ }^{16}$. Moncrieff y cols. (2016) postulan que la ambliaudia puede presentarse como una asimetría mayor de lo esperado en la dominancia de un oído en sujetos con umbrales auditivos normales, pero que puede ser detectada a través de pruebas de escucha dicótica ${ }^{23}$. De esta forma, cualquier evento que cause una pérdida asimétrica de la audición (conductiva o sensorioneural) durante las ventanas críticas del desarrollo cerebral puede dar lugar a dificultades duraderas en entornos auditivos difíciles, en particular en la localización del sonido en el espacio y en la utilización de señales binaurales para procesar el habla en situaciones con altos niveles de ruido ambiental ${ }^{16}$. La causa de la hipoacusia unilateral puede ser de origen congénita (sensorioneural o conductivo) o adquirida. Sin embargo, hay una mayor proporción de estudios de ambliaudia en casos de pérdida auditiva conductiva en comparación a la sensorioneural ${ }^{16}$. Esta diferencia se explica porque en humanos 
resulta más sencillo revertir quirúrgicamente una hipoacusia conductiva como la otitis media con efusión (OME), y porque también es más fácil desarrollar modelos animales de hipoacusia conductiva ${ }^{16}$.

Si bien en forma teórica es posible que la ambliaudia se origine en casos de pérdida auditiva unilateral transitoria, como los provocados por la OME, resulta de importancia destacar que la evidencia al respecto es controversial. Existen estudios que sugieren un vínculo entre la OME y la ambliaudia ${ }^{24}$, mientras que otros no han encontrado asociación entre ambas condiciones $^{25}$. Entre los factores que puede estar influyendo en estos resultados se encuentra el hecho de que el grado de hipoacusia en la OME suele no superar los $40 \mathrm{~dB}$ de pérdida auditiva $^{6,26}$. Dado que la ambliaudia se genera por la pérdida auditiva y no por la OME per se, solo los casos de OME con hipoacusia en grado moderada y persistente podrían estar en riesgo de desarrollar ambliaudia. En este contexto, se hacen necesarias más investigaciones para tener más claridad de la relación entre el grado de severidad de la hipoacusia generada por la OME y la ambliaudia.

Por otra parte, si bien existe menor evidencia al respecto, este fenómeno también puede surgir en pacientes con sordera profunda bilateral y con implante coclear unilateral. El implante coclear bilateral impediría el desarrollo de ambliaudia, y cualquier retraso entre la recepción del primer y segundo dispositivo coclear resulta en un período de pérdida auditiva asimétrica, ya que sólo se estimula un oído. En niños congénitamente sordos, existe evidencia de que pasada una edad crítica el segundo oído implantado tiene muy pocas posibilidades de proveer niveles adecuados de percepción auditiva ${ }^{27}$. Sumado a esto, se han observado mayores beneficios en la comprensión del habla en los individuos con menor desfase temporal entre los dos implantes que, según estos autores, el intervalo de tiempo entre el primer $y$ segundo implante debiera ser menor a 5 años ${ }^{28}$. Además, el uso de implantes bilaterales después de períodos prolongados de implantación unilateral puede provocar alteraciones en la percepción de claves binaurales ${ }^{29}$ y activación cortical lateralizada ${ }^{30}$. Es así como esta evidencia apoya la idea de que, en los períodos críticos del desarrollo auditivo cerebral, la audición asimétrica alteraría de forma prolongada las vías auditivas centrales, generando alteraciones perceptuales permanentes y comprometiendo las posibles intervenciones futuras. Estas alteraciones funcionales y morfológicas estarían asociados tanto a la severidad de la disfunción como a la edad de aparición ${ }^{6,16}$, por lo que un pronto diagnóstico y tratamiento serían claves en su prevención. Esto recalca la importancia clínica de tratar a los recién nacidos con hipoacusia unilateral (de grados moderados o más severos) y de efectuar seguimientos en los casos de hipoacusia unilateral leve, como también el promover el desarrollo de exámenes auditivos universales al ingresar a la etapa escolar $o$ incluso antes en etapas preescolares ${ }^{31}$.

Como consecuencia de estas alteraciones morfofuncionales del sistema nervioso, se espera que en un niño que presente ambliaudia existan dificultades en la localización espacial de sonidos y en la separación entre la señal auditiva de interés y el ruido de fondo. Esto tendría un impacto negativo en las capacidades de comprensión del habla, en especial en ambientes ruidosos. Por lo mismo, se espera observar efectos negativos en el ámbito educacional y en el desarrollo de sus comportamientos sociales. Lo anterior también sugiere que los pacientes con ambliaudia probablemente no manifiesten problemas auditivos (considerando que el evento de hipoacusia unilateral ya está resuelto), sino más bien fatiga y alteraciones psicológicas ${ }^{16}$.

Todo esto pone en evidencia la importancia clínica de la ambliaudia y, también, lo relevante que puede resultar comprender los cambios a largo plazo que ocurren en el sistema nervioso producto de la hipoacusia unilateral. Sin embargo, las bases neurales específicas de esta patología siguen siendo poco claras. En este contexto, mejorar nuestra comprensión acerca del efecto que tiene la hipoacusia unilateral sobre el sistema auditivo podría ayudar en el diseño de nuevas intervenciones terapéuticas como también a predecir de mejor manera el potencial efecto de las estrategias de tratamientos actuales. En los próximos párrafos se revisan datos obtenidos en humanos y en modelos animales que describen las consecuencias neurobiológicas de la deprivación auditiva unilateral. 


\section{Mecanismos neurobiológicos de la ambliaudia}

La ambliaudia se ha estudiado en modelos animales en los que se induce experimentalmente hipoacusia unilateral, incluyendo: (i) la pérdida de audición sensorioneural a través de la ablación coclear unilateral y (ii) la hipoacusia conductiva. Esta última se puede lograr a través de la interrupción de la cadena osicular, y la ligadura o taponamiento del conducto auditivo externo. Las últimas dos estrategias de hipoacusia conductiva resultan completamente reversibles, por lo que imitan de mejor forma las condiciones de la ambliaudia clínica y su posible tratamiento. Sin embargo, la mayor parte de la evidencia que se tiene sobre la plasticidad cerebral con pérdida de audición asimétrica se basa en estudios de ablación coclear unilateral con hipoacusia sensorioneural permanente ${ }^{32}$, lo que no modela de forma adecuada lo que ocurre con el tratamiento de la hipoacusia y la ambliaudia. Por esto, conclusiones basadas en este tipo de modelos deben ser consideradas con cautela. Se ha observado que la hipoacusia unilateral en roedores recién nacidos produce alteraciones en estructuras claves de la vía auditiva (Figura 1). Por ejemplo, la obstrucción crónica del conducto auditivo externo se asocia a la reducción del diámetro de los somas de neuronas del núcleo coclear ${ }^{33}$ y a una maduración incompleta de éste y otras regiones auditivas del tronco encéfalo como el complejo olivar superior y el colículo inferior (CI ${ }^{34}$. El $\mathrm{CI}$ es una estructura clave del sistema auditivo central, en la cual convergen las principales vías auditivas ascendentes y descendentes bilaterales, actuando como una estación integradora. En términos funcionales, el CI ha sido involucrado en múltiples procesos como la detección de tonos y la localización espacial de sonidos. Sus características anátomo-funcionales, como la convergencia de las vías sensoriales binaurales, sugieren que el CI podría estar sujeto a cambios plásticos producto de una pérdida auditiva asimétrica durante períodos críticos de desarrollo.

En modelos animales, se ha visto que la ablación coclear unilateral altera las proyecciones bilaterales hacia el $\mathrm{CI}^{35}$, generando un desbalance en la representación de la vía afectada y la normal. Esto se manifiesta a través de una dominancia o sobrerepresentación de las vías que provienen desde el oído sano. En particular, las aferencias ipsilaterales hacia el CI desde núcleo coclear que aún funciona pueden llegar a duplicarse luego de la ablación neonatal ${ }^{35}$. Además, estas proyecciones ipsilaterales muestran patrones de terminación más amplios dentro del $\mathrm{CI}^{36}$, por lo que podrían ejercer una influencia generalizada en el procesamiento binaural. Los registros fisiológicos en el CI contralateral a la ablación también muestran un aumento en la activación de la estimulación desde el oído intacto ${ }^{37}$, lo cual podría deberse a un aumento de la excitación o a una pérdida de la inhibición contralateral ${ }^{38}$. Por el contrario, las ablaciones bilaterales no provocan cambios en esta relación de proyección ipsi/contralateral ${ }^{39}$, reforzando la idea que dichas alteraciones se deben a la pérdida de audición asimétrica.

La hipoacusia unilateral también provoca alteraciones anátomo-funcionales en regiones corticales asociadas a la vía auditiva. Por ejemplo, en modelos de sordera unilateral en gatos, si bien las respuestas corticales a estímulos no se ven disminuidas en comparación a animales con audición normal se observa una extensa reorganización hemisférica de la dominancia auditiva y de las interacciones corticales binaurales $^{40,41}$. En roedores también se ha descrito una alteración de la actividad de las neuronas de la corteza auditiva primaria, contralateral al oído afectado, siendo más severa en animales que adquirieron la hipoacusia unilateral a menor edad ${ }^{42}$. En la misma línea, estudios de resonancia magnética cerebral en humanos, han asociado a la hipoacusia unilateral con atrofias y cambios funcionales en áreas corticales, incluyendo cortezas involucradas en memoria, atención y funciones ejecutivas ${ }^{43-46}$. Si bien estos resultados evidencian los efectos que tiene la hipoacusia unilateral sobre las vías auditivas y el sistema nervioso central, como se mencionó anteriormente, la mayoría de estas investigaciones no pueden considerarse modelos adecuados de ambliaudia. Idealmente, un modelo experimental de esta condición debería contemplar el estudio de los cambios anátomo-funcionales de los circuitos auditivos en animales con antecedentes de pérdida auditiva unilateral, pero con umbrales auditivos normales en el momento de la prueba. A pesar de que existen muchos menos estudios que 


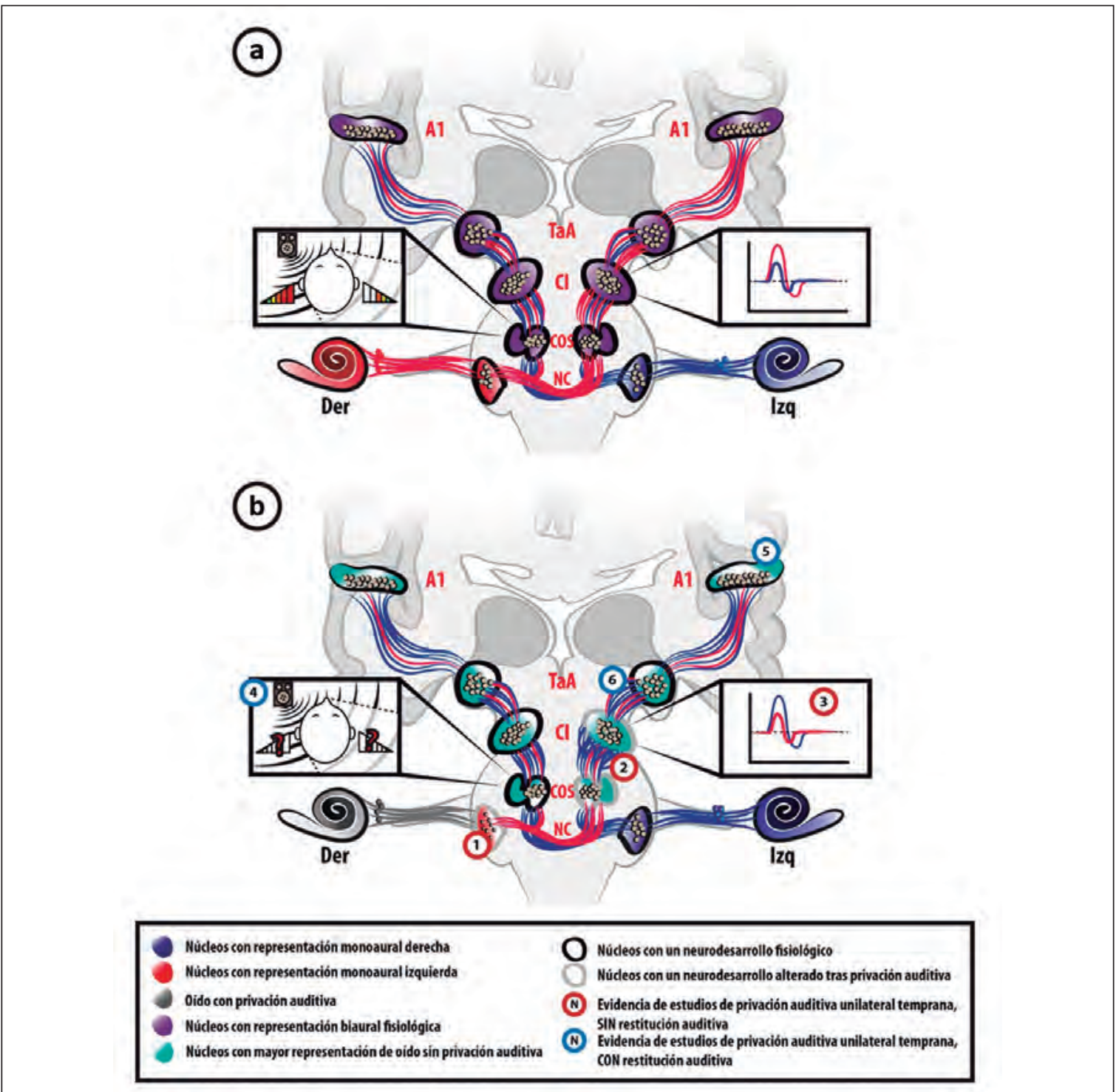

Figura 1. Vías auditivas centrales en audición normal (a) y en ambliaudia (b). a: Umbrales auditivos normales bilaterales. Se grafican la cóclea y núcleo coclear derecho en rojo, los izquierdos en azul. El resto de la vía se grafica en morado representando el aporte biaural en cada una de las regiones de la vía auditiva. Los mapas tonotópicos se representan por la coloración en degradado dentro de cada región. Los cuerpos neuronales se grafican como círculos en cada área. Las fibras se representan como líneas rojas o azules representando la inervación relativa desde cada núcleo coclear al resto de la vía. En los casilleros se representa a la izquierda la codificación a nivel del complejo olivar superior de diferencias interaurales de intensidad de sonido, a la izquierda la respuesta electrofisiológica a nivel del colículo inferior, destacando una mayor respuesta la estimulación del oído contralateral, representado por el gráfico azul, versus la actividad del oído ipsilateral representado en color rojo. b: Ambliaudia del sistema auditivo central por hipoacusia unilateral del oído derecho. La cóclea y núcleos cocleares se grafican igual que en el panel A. Se representan alteraciones secundarias a privación auditiva temprana de oído derecho (rojo). Note que el degradado de color dentro de cada región es diferente a los graficados en el panel A, representando la alteración de los mapas tonotópicos secundarios a la privación auditiva unilateral (5). A nivel del núcleo coclear derecho se observa una disminución del diámetro de los somas neuronales (1). Existe una mayor representación del oído intacto a nivel de las diferentes áreas a lo largo de la vía, graficado por un mayor número de fibras desde el oído izquierdo (6) y por patrones de terminación más amplio de las aferencias hacia el colículo inferior (2). Existe una alteración en la codificación de intensidad interaural representada en el casillero izquierdo (4) y un aumento de la actividad electrofisiológica en respuesta a la estimulación del oído intacto (ipsilateral) (3). NC: núcleo coclear, COS: complejo olivar superior, Cl: colículo inferior, CGM: cuerpo geniculado medial, A1: corteza auditiva primaria. 
cumplan esta condición, los que lo hacen también han encontrado alteraciones en el balance biaural de los campos tonotópicos de neuronas del CI y de la corteza auditiva primaria, incluso cuando el oído que estuvo desprovisto de desarrollo actualmente tiene umbrales auditivos normales ${ }^{47}$. En particular, se han observado distorsiones en los mapas tonotópicos de la vía auditiva, donde se debilita la representación del oído privado, se fortalece la representación del oído normal y se interrumpe la integración biaural de las diferencias interaurales de intensidad y latencia ${ }^{30,47}$. Registros con arreglos de multielectrodos implantados en el CI y en la corteza auditiva primaria obtenidos tras la corrección de una hipoacusia conductiva asimétrica prolongada muestran una reducción en las representaciones neurales del oído ocluido, la que perdura a pesar de tener una audición periférica normal ${ }^{19,47}$. Este tipo de alteraciones pueden persistir hasta años después del uso de implantes cocleares bilaterales ${ }^{30}$. En relación con esto, un estudio reciente evaluó la actividad cortical en niños con pérdida de audición asimétrica que utilizaban dispositivos auditivos bimodales ${ }^{48}$. De forma similar a los casos descritos, se encontró que la audición asimétrica prolongada altera las cortezas auditivas creando una preferencia por el mejor oído. Se observó que esta preferencia por el oído implantado ocurría con el uso continuo del implante, cuando la audición era deficiente en el oído no implantado. De hecho, las alteraciones no se manifestaban si es que de forma temprana había una estimulación biaural equilibrada.

Sumado a todo lo anterior, es importante mencionar que cualquier discusión sobre la plasticidad auditiva y cerebral no puede dejar de lado la contribución de las numerosas proyecciones descendentes de los centros auditivos superiores a los núcleos inferiores ${ }^{49}$. Aunque el papel de estos circuitos en el procesamiento auditivo no es del todo conocido, existe evidencia de que juegan rol importante en el ajuste de las señales auditivas ascendentes en función de la memoria, el contexto y las expectativas ${ }^{50}$. Se ha observado que el sistema eferente auditivo permite reducir la sensibilidad de los receptores y amortiguar el ruido de fondo para producir un efecto antienmascaramiento y mejorar la discriminación de las señales externas ${ }^{51}$.
Además, ha sido involucrado como un filtro biológico en tareas de atención selectiva ${ }^{52,53}$. En consecuencia, este sistema sería esencial para la audición selectiva y para escucha en ambientes ruidosos. En el caso de la hipoacusia asimétrica, hay evidencia de que las proyecciones descendentes facilitan la habilidad de volver a aprender a localizar sonidos basados en las señales biaurales alteradas introducidas por la oclusión del conducto auditivo externo ${ }^{54}$. Si tales adaptaciones permanecen después de que se restaura la audición equilibrada, las señales auditivas eferentes se verían afectadas y podrían estar contribuyendo al proceso de la ambliaudia. Por lo tanto, alteraciones en el sistema auditivo eferente también podrían estar contribuyendo a la fisiopatología de la ambliaudia, tanto la percepción biaural alterada como la dificultad de procesar las señales del habla en situaciones de altos niveles de ruido de fondo. Sin embargo, existe poca evidencia experimental que permita inferir cómo el sistema eferente auditivo se ve afectado por la pérdida auditiva asimétrica, en especial si ésta ocurre durante el desarrollo ${ }^{55}$.

\section{Conclusión}

Para concluir, se debe recalcar que aún existen muchas preguntas abiertas respecto al fenómeno de la ambliaudia. Si bien sabemos que esta parece ser producto de una señalización aferente interrumpida de la vía auditiva central, no es claro cuál es el umbral auditivo necesario de pérdida en decibeles para causar defectos duraderos, ni tampoco las ventanas exactas de desarrollo crítico en seres humanos. Además, se desconoce si las diferentes etiologías de privación (pérdida auditiva sensorioneural y conductiva) cambian las características y secuelas de este diagnóstico. Tampoco conocemos a cabalidad el impacto a nivel cerebral de esta condición, en especial los asociados a las vías eferentes auditivas y a regiones cerebrales más allá de las auditivas. Todo esto influye en que hoy, el diagnóstico y la cuantificación de la ambliaudia siguen siendo controversiales y difíciles de definir. Por todo esto, se hace necesario el desarrollo de investigaciones diseñadas específicamente para el estudio de la ambliaudia, que permitan 
comprender de mejor manera las implicancias clínicas de este fenómeno y así facilitar las decisiones de tratamiento con respecto al manejo de la pérdida auditiva asimétrica.

\section{Bibliografía}

1. Bravo AR, Krefft MM, Gómez YF, et al. Indicadores de calidad del Programa de Detección Precoz de Hipoacusia Permanente del Hospital Padre Hurtado. Rev Otorrinolaringol Cir Cabeza Cuello 2017;77:117123.

2. Nazar G, Goycoolea M, Godoy JM, et al. Evaluación auditiva neonatal universal: Revisión de 10.000 pacientes estudiados. Rev Otorrinolaringol Cir Cabeza Cuello 2009;69:93-102.

3. Finitzo T, Albright K, O’Neal J. The newborn with hearing loss: Detection in the nursery. Pediatrics 1998 ; 102:1452-1460.

4. Fortnum HM, Summerfield AQ, Marshall DH, et al. Prevalence of permanent childhood hearing impairment in the United Kingdom and implications for universal neonatal hearing screening: Questionnaire based ascertainment study. Br Med J 2001;323:536-539.

5. Eiserman WD, Hartel DM, Shisler L, et al. Using otoacoustic emissions to screen for hearing loss in early childhood care settings. Int J Pediatr Otorhinolaryngol 2008;72:475-482.

6. Whitton JP, Polley DB. Evaluating the perceptual and pathophysiological consequences of auditory deprivation in early postnatal life: A comparison of basic and clinical studies. J Assoc Res Otolaryngol 2011;12:535-546.

7. Fitzpatrick EM, Whittingham JA, Durieux-Smith A. Mild bilateral and unilateral hearing loss in childhood: A 20-year view of hearing characteristics, and audiologic practices before and after newborn hearing screening. Ear Hear 2014;35:10-18.

8. Gordon K, Henkin Y, Kral A. Asymmetric Hearing During Development: The Aural Preference Syndrome and Treatment Options. Pediatrics 2015;136:141-153.

9. Fitzpatrick EM, Durieux-Smith A, Whittingham J. Clinical practice for children with mild bilateral and unilateral hearing loss. Ear Hear 2010;31:392-400.

10. Vila PM, Lieu JEC. Asymmetric and unilateral hearing loss in children. Cell Tissue Res 2015; 361:271278.

11. Bess $\mathrm{F}$, Tharpe AM. Unilateral hearing impairment in children. Pediatrics 1984;74:206-16.

12. Lieu JEC. Speech-language and educational consequences of unilateral hearing loss in children. Arch Otolaryngol Head Neck Surg 2004;130:524-530.

13. Wie OB, Pripp AH, Tvete O. Unilateral deafness in adults: Effects on communication and social interaction. Ann Otol Rhinol Laryngol 2010;119:772781.

14. Lieu JEC, Tye-Murray N, Fu Q. Longitudinal study of children with unilateral hearing loss. Laryngoscope 2012;122:2088-95.

15. Lieu JEC. Unilateral hearing loss in children: Speechlanguage and school performance. B-ENT 2013;107115.

16. Kaplan AB, Kozin ED, Remenschneider A, et al. Amblyaudia. Otolaryngol Head Neck Surg 2016;154:247-255.

17. King AJ, Moore DR. Plasticity of auditory maps in the brain. Trends Neurosci 1991;14:31-37.

18. Knudsen EI. Sensitive periods in the development of the brain and behavior. J Cogn Neurosci 2004;16:14121425.

19. Polley DB, Thompson JH, Guo W. Brief hearing loss disrupts binaural integration during two early critical periods of auditory cortex development. Nat Commun 2013;4:1-13. Disponible en https://doi. org/10.1038/ncomms3547.

20. Wilmington D, Gray L, Jahrsdoerfer R. Binaural processing after corrected congenital unilateral conductive hearing loss. Hear Res 1994;74:99-114.

21. Breier JI, Hiscock M, Jahrsdoerfer RA, et al. Ear advantage in dichotic listening after correction for early congenital hearing loss. Neuropsychologia 1998;36:209-216.

22. Johnstone PM, Nábělek AK, Robertson VS. Sound localization acuity in children with unilateral hearing loss who wear a hearing aid in the impaired ear. J Am Acad Audiol 2010;21:522-534.

23. Moncrieff D, Keith W, Abramson M, Swann A. Diagnosis of amblyaudia in children referred for auditory processing assessment. Int J Audiol 2016;55:333-345.

24. Hogan SCM, Moore DR. Impaired binaural hearing in children produced by a threshold level of middle ear disease. J Assoc Res Otolaryngol 2003;4:123-129.

25. Roberts JE, Burchinal MR, Zeisel SA. Otitis Media in Early Childhood in Relation to Children's SchoolAge Language and Academic Skills. Pediatrics 2002;110:696-706.

26. Roberts J, Hunter L, Gravel J, et al. Otitis media, hearing loss, and language learning controversies and current research. J Dev Behav Pediatr 2004;25:110122.

27. Graham J, Vickers D, Eyles J, et al. Bilateral sequential cochlear implantation in the congenitally deaf child: Evidence to support the concept of a "critical age" after which the second ear is less likely to provide an adequate level of speech perception on its own. Cochlear Implants Int 2009; 10:119-141.

28. Illg A, Giourgas A, Kral A, et al. Speech comprehension in children and adolescents after 
sequential bilateral cochlear implantation with long interimplant interval. Otol Neurotol 2013;34:682-689.

29. Gordon KA, Valero J, Van Hoesel R, et al. Abnormal timing delays in auditory brainstem responses evoked by bilateral cochlear implant use in children. Otol Neurotol 2008;29:193-198.

30. Gordon KA, Wong DDE, Papsin BC. Bilateral input protects the cortex from unilaterally-driven reorganization in children who are deaf. Brain 2013;136:1609-1625.

31. Prieve BA, Schooling T, Venediktov R, Franceschini N. An Evidence-Based Systematic Review on the Diagnostic Accuracy of Hearing Screening Instruments for Preschool-and School-Age Children. Am J Audiol 2015;24:250-267. Disponible en https:// doi.org/10.1044/2015_AJA-14-0065.

32. Moore DR, King AJ. Plasticity of Binaural Systems. Plast Audit Syst 2004;96-172.

33. Coleman JR, O'Connor P. Effects of monaural and binaural sound deprivation on cell development in the anteroventral cochlear nucleus of rats. Exp Neurol 1979;64:553-566.

34. Webster D, Webster M. Effects of neonatal conductive hearing loss on brain stem auditory nuclei. Ann Otol Rhinol Laryngol 1979;88:684-8.

35. Nordeen KW, Killackey HP, Kitzes LM. Ascending projections to the inferior colliculus following unilateral cochlear ablation in the neonatal gerbil, Meriones unguiculatus. J Comp Neurol 1983;214:144153.

36. Moore DR, Kitzes LM. Projections from the cochlear nucleus to the inferior colliculus in normal and neonatally cochlea-ablated gerbils. J Comp Neurol 1985;240:180-95.

37. McAlpine D, Martin RL, Mossop JE, et al. Response Properties of Neurons in the Inferior Colliculus of the Monaurally Deafened Ferret to Acoustic Stimulation of the Intact Ear. J Neurophysiol 1997;78:767-79.

38. Popelar J, Erre J, Aran J, et al. Plastic changes in ipsi-contralateral differences of auditory cortex and inferior colliculus evoked potentials after injury to one ear in the adult guinea pig. Hear Res 1994;72:12534.

39. Moore DR. Auditory brainstem of the ferret: bilateral cochlear lesions in infancy do not affect the number of neurons projecting from the cochlear nucleus to the inferior colliculus. Dev Brain Res 1990;54:125-30.

40. Kral A, Tillein J, Hubka P, et al. Spatiotemporal patterns of cortical activity with bilateral cochlear implants in congenital deafness. J Neurosci 2009;29:811-27.

41. Tillein J, Hubka P, Kral A. Monaural Congenital Deafness Affects Aural Dominance and Degrades Binaural Processing. Cereb Cortex 2016;26:1762-77.

42. Wang X, Liu J, Zhang J. Chronic unilateral hearing loss disrupts neural tuning to sound-source azimuth in the rat primary auditory cortex. Front Neurosci 2019;13:1-18.

43. Tibbetts K, Ead B, Umansky A, et al. Interregional brain interactions in children with unilateral hearing loss. Otolaryngol Head Neck Surg 2011;144:602-611.

44. Wang X, Fan Y, Zhao F, et al. Altered regional and circuit resting-state activity associated with unilateral hearing loss. PLoS One 2014;9:e96126. Disponible en https://doi.org/10.1371/journal.pone.0096126.

45. Zeng C, Yang Z, Shreve L, et al. Somatosensory Projections to Cochlear Nucleus Are Upregulated after Unilateral Deafness. J Neurosci 2012;32:1579115801. Disponible en https://doi.org/10.1523/ JNEUROSCI.2598-12.2012.

46. Yang M, Chen HJ, Liu B, et al. Brain structural and functional alterations in patients with unilateral hearing loss. Hear Res 2014;316:37-43. Disponible en https://doi.org/10.1016/j.heares.2014.07.006.

47. Popescu MV, Polley DB. Monaural Deprivation Disrupts Development of Binaural Selectivity in Auditory Midbrain and Cortex. Neuron 2010;65:718731. Disponible en https://doi.org/10.1016/j. neuron.2010.02.019.

48. Polonenko MJ, Papsin BC, Gordon KA. Delayed access to bilateral input alters cortical organization in children with asymmetric hearing. NeuroImage Clin 2018;17:415-425. Disponible en https://doi. org/10.1016/j.nicl.2017.10.036.

49. Malmierca M, Ryugo D. Descending Connections of Auditory Cortex to the Midbrain and Brain Stem. In: Audit Cortex. New York: Springer, 2011. pp. 189-208.

50. Bajo VM, King AJ. Cortical modulation of auditory processing in the midbrain. Front Neural Circuits 2013;6:1-12.

51. Guinan JJ. Cochlear efferent innervation and function. Curr Opin Otolaryngol Head Neck Surg 2010;18:447-453.

52. Délano PH, Elgueda D, Hamame CM, et al. Selective attention to visual stimuli reduces cochlear sensitivity in chinchillas. J Neurosci 2007;27:4146-4153.

53. Terreros G, Jorratt P, Aedo C, et al. Selective Attention to Visual Stimuli Using Auditory Distractors Is Altered in Alpha-9 Nicotinic Receptor Subunit Knock-Out Mice. J Neurosci 2016;36:71987209. Disponible en https://doi.org/10.1523/ JNEUROSCI.4031-15.2016.

54. Bajo VM, Nodal FR, Moore DR, et al. The descending corticocollicular pathway mediates learning-induced auditory plasticity. Nat Neurosci 2010;13:253-260. Disponible en https://doi.org/10.1038/nn.2466.

55. Lesicko AMH, Llano DA. Impact of peripheral hearing loss on top-down auditory processing. Hear Res 2017;343:4-13. Disponible en https://doi. org/10.1016/j.heares.2016.05.018. 